\title{
Avaliação da formação de smear layer no preparo químico mecânico realizado com solução ou gel de hipoclorito de sódio a $2,5 \%$
}

\section{Evaluation of smear layer formation in mechanical chemical preparation performed with $2.5 \%$ sodium hypochloride solution or gel}

\author{
Luciana Batista Luz ${ }^{1}$ (D), Ruth Marlene Campomanes Santana ${ }^{2}$ (D), Amanda Prates ${ }^{3}$ (D), Julia Froehlich ${ }^{4}$ (D) Tiago André Fontoura de \\ Melo $^{5}$ (D), Francisco Montagner ${ }^{5}$ (D) , Simone Bonato Luisi ${ }^{5}$ (D)
}

1. Doutoranda em Odontologia pela Universidade Federal do Rio Grande do Sul (UFRGS), Porto Alegre, RS, Brasil. 2. Docente, Laboratório de Materiais Poliméricos - LAPOL - Departamento de Engenharia de Materiais da Universidade Federal do Rio Grande do Sul (UFRGS), Porto Alegre, RS, Brasil. 3. Acadêmica de Odontologia, Faculdade de Odontologia da Universidade Federal do Rio Grande do Sul (UFRGS), Porto Alegre, RS, Brasil. 4. Aluna do Curso de Especialização em Endodontia, Faculdade de Odontologia da Universidade Federal do Rio Grande do Sul (UFRGS), Porto Alegre, RS, Brasil. 5. Docente, Departamento de Odontologia Conservadora, Faculdade de Odontologia da Universidade Federal do Rio Grande do Sul (UFRGS), Porto Alegre, RS, Brasil.

\section{Resumo}

Introdução: a formação de smear layer durante o preparo dos canais radiculares com o uso de hipoclorito de sódio solução já foi amplamente estudada; entretanto, na apresentação em gel não existem relatos na literatura. Objetivo: avaliar, em MEV, a formação de smear layer durante o preparo endodôntico, utilizando o $\mathrm{NaOCl}$ nas formas líquida e gel, associado ou não ao EDTA. Métodos: sessenta raízes palatinas de molares superiores ou distais de inferiores, com comprimento radicular padronizado em $15 \mathrm{~mm}$, foram utilizadas. As amostras foram divididas em quatro grupos controle com $\mathrm{n}=5$ cada (soro fisiológico ou base gel, associados ou não ao EDTA) e quatro grupos testes com $n=10$ cada, de acordo com a substância irrigadora empregada durante o preparo (solução ou gel de $\mathrm{NaOCl}$ a 2,5\%, associados ou não ao EDTA). Os canais foram modelados e posteriormente clivados para análise em MEV (ampliação de 2000x). Imagens de eletromicrografias foram obtidas nos terços cervical, médio e apical. A presença e as características da smear layer formadas foram categorizadas. As imagens foram analisadas por dois avaliadores, previamente, calibrados. Após a análise de concordância $($ Kappa = 0,806) dos escores, os dados foram tratados com o uso do teste Kruskall-Wallis, complementado pelo teste de Comparações Múltiplas SNK. 0 nível de significância foi de 5\%. Resultados: a utilização do soro fisiológico e do $\mathrm{NaOCl}$ líquido, associados à irrigação final com EDTA, apresentou os melhores resultados de limpeza. Com o uso do $\mathrm{NaOCl}$ gel e da base gel, sem associação ao EDTA, houve uma maior quantidade de resíduos sobre as paredes dentinárias. Além disso, independentemente da substância irrigadora testada, pode-se observar que houve uma melhor capacidade de remoção da smear layer no terço cervical em relação ao apical. Conclusões: observou-se formação de smear layer após o preparo do canal radicular associado ao $\mathrm{NaOCl}$ líquido ou gel. A aplicação de EDTA promoveu a remoção da smear layer produzida durante o preparo do canal.

Palavras-chave: Endodontia. Preparo de canal radicular. Hipoclorito de sódio. Microscopia Eletrônica de Varredura.

\begin{abstract}
Introduction: the formation of smear layer during the preparation of root canals with the use of sodium hypochlorite solution has been widely studied; however, in the gel presentation there aren't reports in the literature. Objective: to evaluate, in SEM, the formation of smear layer during endodontic preparation, using $\mathrm{NaOCl}$ in liquid and gel forms, associated or not with EDTA. Methods: sixty palatal roots of maxillary molars or distal roots of lower molars, with a root length of $15 \mathrm{~mm}$, were used. The samples were divided into four control groups with $\mathrm{n}=5$ each (saline or gel base, associated or not with EDTA $)$ and four test groups $(\mathrm{n}=10 \mathrm{each})$ according to the irrigation substance used during the preparation $(2.5 \% \mathrm{NaOCl}$ solution or gel, associated or not with EDTA). The root canals were instrumented and later split for SEM analysis (2000x magnification). Electromicrographic images were obtained from the cervical, middle and apical thirds. The presence and characteristics of the smear layer formed were categorized. The images were analyzed by two previously calibrated evaluators. After the Kappa analysis (0.806), the data were treated using the Kruskall-Wallis test, complemented by the SNK Multiple Comparison Test. The level of significance was 5\%. Results: the use of saline and liquid NaOCl, associated with final rinsing with EDTA, showed the best cleaning results. With the use of $\mathrm{NaOCl}$ gel and gel base, with no EDTA association, there was a greater amount of residues on the dentin surfaces. In addition, despite the irrigation substance tested, it can be observed that there was a better capacity of removal of the smear layer in the cervical third in relation to the apical. Conclusion: root canal instrumentation associated with $\mathrm{NaOCl}$ solution or gel EDTA produced smear layer. Smear layer was removed with the use of EDTA as a final flushing.
\end{abstract}

Key words: Endodontics. Root canal preparation. Sodium hypochlorite. Scanning Electron

\section{INTRODUÇÃO}

Os microrganismos e seus produtos metabólicos são considerados os principais responsáveis pelo desenvolvimento e pela perpetuação das doenças pulpares e periapicais.
Desse modo, a desinfecção do sistema de canais radiculares é parte essencial para que se atinja o sucesso do tratamento endodôntico. Embora existam variedades de técnicas e 
160 Formação de smear layer com uso da solução ou gel de hipoclorito de sódio no preparo

métodos de instrumentação, é frequente a permanência de resíduos, microrganismos, restos de tecido pulpar e raspas de dentina, após a finalização do preparo químico mecânico ${ }^{1,2}$. Remanescentes de tecido necrótico no interior do canal radicular podem ser uma fonte de nutrição para os microrganismos sobreviventes ${ }^{3}$.

Em função da complexidade anatômica do sistema de canais radiculares, aproximadamente $50 \%$ das paredes dos canais permanecem não instrumentadas durante o preparo, o que resulta em uma limpeza insuficiente ${ }^{4}$. Por essa razão, deve-se combinar o uso de substâncias químicas ao preparo mecânico, a fim de potencializar e complementar o processo de desinfecção. Os agentes químicos selecionados como substâncias irrigadoras devem apresentar algumas propriedades principais, como atividade antimicrobiana, poder de dissolução de matéria orgânica, baixa tensão superficial, ação lubrificante e devem ser atóxicos para tecidos periapicais ${ }^{5}$.

A substância irrigadora mais amplamente utilizada na endodontia é o hipoclorito de sódio $(\mathrm{NaOCl})$ nas concentrações de $0,5 \%$ a $6,0 \%$, devido à sua ação antimicrobiana, pela formação de ácido hipocloroso e posterior liberação de cloro livre e sua capacidade de dissolução da matéria orgânica por meio da quebra de proteínas e aminoácidos ${ }^{6,7}$.

No entanto, existem algumas desvantagens quando do emprego do $\mathrm{NaOCl}$ como substância irrigadora. Além do odor e do sabor desagradável, a atividade citotóxica dessa substância pode causar lesões agudas, caso atinja a região periapical. Em contato com tecidos vitais, o $\mathrm{NaOCl}$ rapidamente oxida a matéria orgânica, levando a uma rápida hemólise e ulceração, inibição da migração de neutrófilos e destruição de células endoteliais e fibroblastos ${ }^{8,9}$.

A extrusão de $\mathrm{NaOCl}$ para os tecidos periapicais, durante o tratamento endodôntico, provoca sintomas agudos imediatos, potencialmente graves e com riscos de sequelas ${ }^{10-12}$. Segundo o estudo de Kleier et al. (2008), sobre a ocorrência de acidentes com $\mathrm{NaOCl}$ por endodontistas da American Board, quase metade dos profissionais relataram a ocorrência de pelo menos um acidente com $\mathrm{NaOCl}$ durante o tratamento endodôntico ${ }^{13}$.

Por esta razão, muitas tentativas têm sido feitas para encontrar outras formulações de substâncias irrigadoras eficientes, com uma elevada ação antimicrobiana, que apresentem baixo poder de toxicidade ${ }^{14}$.

As substâncias utilizadas durante o preparo químico mecânico são geralmente líquidas. A utilização de uma substância irrigadora mais viscosa pode ser interessante para evitar ou reduzir o risco da sua penetração nos tecidos periapicais ${ }^{5,14}$.

Ferraz et al. (2001) mostraram que a clorexidina quando utilizada na forma de gel a $2 \%$, devido à sua viscosidade, promove uma melhor ação no interior do canal radicular, removendo dentina e tecidos remanescentes quando associado ao ato de instrumentação. Essa condição permite que a clorexidina ultrapasse a sua incapacidade de dissolver tecidos orgânicos ${ }^{14}$. Considerando os aspectos levantados, o $\mathrm{NaOCl}$, na forma de gel, pode ser uma boa opção como auxiliar químico no preparo dos canais radiculares. $O$ produto preservaria as suas propriedades químicas e biológicas associadas à ação mecânica do gel. Além disso, segundo o estudo de Zand et al. (2010), o uso de gel de $\mathrm{NaOCl}$ pode ser eficaz, também, na remoção da smear layer 9 .

Para Poggio et al. (2010), a utilização de bases viscosas nas substâncias irrigadoras podem deixar resíduos, pouco solúveis em água, aderidos às paredes dentinárias, o que prejudica sua remoção ${ }^{5}$.

Dessa forma, o presente estudo tem como objetivo avaliar a formação de smear layer com a realização do preparo químicomecânico, utilizando o $\mathrm{NaOCl}$ nas formas líquida e gel, associado ou não à irrigação final com EDTA.

\section{MÉTODOS}

O presente estudo foi aprovado pelo Comitê de Pesquisa da Faculdade de Odontologia da UFRGS e pelo Comitê de Ética em Pesquisa da mesma instituição (Protocolo CAAE 68435317.1.0000.5347).

\section{Preparação do gel de hipoclorito de sódio a 2,5\%}

A base do gel foi produzida a partir de $10 \%$ de gel, com viscosidade de 450 centipoises, similar ao gel de clorexidina a $2 \%$ (Essential Pharma, Itapetininga, São Paulo, Brasil), a 25,9ำ com um torque de $17,2 \%$ a 10 rotações por minuto (RPM). A base utilizada é um polímero sintético, solúvel em água, transparente, biocompatível, biologicamente inerte, não mutagênico, nem citotóxico, pois não tem atividade carcinogênica ou antigênica.

O gel ou solução de hipoclorito de sódio a $2,5 \%$ foi produzido pela fórmula: $\mathrm{C} 1 . \mathrm{V} 1=\mathrm{C} 2 . \mathrm{V} 2$ (concentração1 $\mathrm{X}$ volume1 = concentração $2 \mathrm{X}$ volume2), a partir de uma solução concentrada de $\mathrm{NaOCl}$, previamente denominada (Mediquímica Indústria Ltda., Porto Alegre, Brasil).

\section{Preparo das amostras}

Sessenta raízes distais de molares inferiores ou palatinas de molares superiores humanos foram divididas em quatro grupos-teste (solução de $\mathrm{NaOCl}$ a 2,5\% ou gel, associado ou não à solução EDTA a 17\%) e em quatro grupos-controle (soro fisiológico ou gel, associado ou não ao EDTA a 17\%). Para padronizar o comprimento em $15 \mathrm{~mm}$, as coroas dos dentes foram removidas na junção amelocementária.

A exploração inicial do canal radicular foi realizada com um instrumento endodôntico manual de aço inoxidável tipo K \#15 (Dentsply/Maillefer, Ballaigues, Suíça), penetrando gradualmente até que a ponta do instrumento ficasse justaposta 
à saída foraminal. Então, $1 \mathrm{~mm}$ foi deduzido, determinando o comprimento de trabalho (CT). O diâmetro na região apical dos canais foi padronizado com o instrumento tipo $\mathrm{K} \# 20$, e canais com diâmetros menores ou maiores que este foram excluídos do estudo.

Previamente, a realização dos protocolos de tratamento, com o auxílio de um disco diamantado dupla face (KG Sorensen Indústria e Comércio Ltda., Barueri, São Paulo, Brasil), em baixarotação, foram realizados sulcos de orientação na face externa das raízes, no sentido longitudinal das faces vestibular e lingual, com o cuidado para não atingir o interior do canal radicular. Esse procedimento foi realizado a fim de facilitar a clivagem das amostras após a realização dos protocolos de tratamento nos grupos experimentais.

Inicialmente, os canais foram explorados no CT com instrumentos endodônticos tipo K \#10 e \#15 (Dentsply/Maillefer, Ballaigues, Suíça).

Para o preparo dos canais radiculares, foram utilizados instrumentos endodônticos tipo $K$, primeira e segunda séries, seguindo a técnica de step-back com recuo progressivo e programado. Como instrumento memória foi padronizado o \#35, o escalonamento foi realizado até o instrumento \#50.

Antes do uso de cada instrumento, o canal radicular foi preenchido em toda a sua extensão com irrigantes testes ou controles. Após o uso de cada instrumento, foi feita a irrigação do canal radicular, a $4 \mathrm{~mm}$ aquém do $\mathrm{CT}$, com $2 \mathrm{~mL}$ de soro fisiológico visando à remoção de resíduos do material excisado e do irrigante.

Metade das amostras recebeu toalete final de solução de EDTA a 17\% (lodontec Indústria e Comércio de Produtos Odontológicos Ltda., Porto Alegre, Brasil) por 3 minutos. O quelante foi colocado no interior do canal radicular, agitado com o instrumento memória, permanecendo por 3 minutos.

\section{Análise da formação da smear layer}

Após a realização dos protocolos, as amostras foram clivadas e analisadas em Microscopia Eletrônica de Varredura (JEOL JSM 6060, JEOL, Akishima, Japão) com um aumento de 2000 vezes, seguindo o protocolo descrito por Ahmetoglu et al. (2014) $)^{15}$.

Micrografias eletrônicas foram realizadas em três zonas de cada hemissecção dentária. A zona mais cervical localizava-se nos $3 \mathrm{~mm}$ iniciais do canal radicular, a região mais apical nos $3 \mathrm{~mm}$ finais e a região do meio localizada no espaço entre as duas medidas anteriores. Todas as micrografias foram salvas em arquivo digital (CD-ROM, CD-R Maxell ${ }^{\circledR}$ ) com resolução (600 dpi). As imagens foram codificadas com o objetivo de cegar os grupos.

A análise das imagens das micrografias foi realizada por dois avaliadores previamente calibrados, utilizando como critério os escores definidos pelo estudo anterior (Quadro 1$)^{16}$.

Previamente à análise estatística, foi determinada a concordância entre os escores atribuídos às amostras pelos dois avaliadores que participaram deste estudo, por meio do Teste Kappa. O valor Kappa obtido foi de 0,806.

Quadro 1. Tabela descritiva dos escores.

\begin{tabular}{|ll|}
\hline Escores & Descrição dos escores \\
\hline Escore 0 & $\begin{array}{l}\text { Ausência de smear layer. Túbulos dentinários } \\
\text { abertos e livres de debris. }\end{array}$ \\
Escore 1 & $\begin{array}{l}\text { Smear layer moderada. Contorno dos túbulos } \\
\text { dentinários visíveis ou parcialmente preenchidos } \\
\text { com detritos. }\end{array}$ \\
Escore 2 & $\begin{array}{l}\text { Espessa camada de smear layer. Túbulos } \\
\text { dentinários obliterados. }\end{array}$ \\
\hline
\end{tabular}

\section{Análise estatística}

A análise estatística foi realizada com o apoio do Statistical Package for the Social Sciences - SPSS versão 22.0 (SPSS Inc, Chicago, IL). A hipótese nula foi que não há diferença estatística na formação da smear layer promovida pelos auxiliares químicos testados (teste de Kruskal Wallis, SNK Multiple Comparison Test).

\section{RESULTADOS}

Os resultados para os grupos experimentais em cada terço radicular estão expressos na tabela 1 . Comparando os grupos da mesma substância irrigadora, com e sem associação de EDTA, pode-se verificar que os melhores resultados de limpeza da parede dentinária e a presença de túbulos dentinários abertos nos três terços do canal radicular estavam nos grupos que utilizavam o EDTA. A única exceção foi nos grupos de controle de gel. Observou-se, também, que nos grupos que utilizaram soro fisiológico ou solução de $\mathrm{NaOCl}$, associados ao toalete final com EDTA, obtiveram os melhores resultados de limpeza nos três terços radiculares em relação aos demais grupos testados. Os piores resultados, com diferença estatisticamente significante, foram verificados nos grupos que utilizaram o $\mathrm{NaOCl}$ gel e os grupos controle com gel e soro fisiológico, todos sem associação com EDTA. A comparação entre os três terços radiculares em cada um dos grupos experimentais é mostrada também na tabela 1 . Pode-se verificar que o terço cervical apresentou os melhores resultados de limpeza da parede dentinária e a presença de túbulos dentinários abertos em todos os grupos testados, quando comparados ao apical terço do canal (Figura 1), com diferença estatística significativa nos grupos soro fisiológico (GS), Gel + EDTA (GGE), Solução de $\mathrm{NaOCl}+\operatorname{EDTA}(\mathrm{GHLE}), \mathrm{Gel}$ de $\mathrm{NaOCl}(\mathrm{GHG})$ e Gel de $\mathrm{NaOCl}+$ EDTA (GHGE). 
162 Formação de smear layer com uso da solução ou gel de hipoclorito de sódio no preparo

Tabela 1. Grau de presença de smear layer formada entre os grupos experimentais em relação a cada terço radicular.

\begin{tabular}{|c|c|c|c|c|}
\hline \multirow[t]{2}{*}{ Grupos Experimentais } & \multirow[t]{2}{*}{ Escores } & \multicolumn{3}{|c|}{ Terços radiculares } \\
\hline & & Terço Cervical & Terço Médio & Terço Apical \\
\hline \multirow[t]{4}{*}{ Soro fisiológico } & 0 & 1 & 0 & 0 \\
\hline & 1 & 3 & 1 & 0 \\
\hline & 2 & 1 & 4 & 5 \\
\hline & & $\mathrm{AB}$ a & $A a b$ & $A b$ \\
\hline \multirow[t]{4}{*}{ Soro fisiológico + EDTA } & 0 & 5 & 4 & 4 \\
\hline & 1 & 0 & 1 & 1 \\
\hline & 2 & 0 & 0 & 0 \\
\hline & & $\mathrm{Aa}$ & $\mathrm{B} \mathrm{a}$ & $\mathrm{Ca}$ \\
\hline \multirow[t]{4}{*}{ Gel } & 0 & 0 & 1 & 0 \\
\hline & 1 & 3 & 1 & 0 \\
\hline & 2 & 2 & 3 & 5 \\
\hline & & $\mathrm{Ba}$ & $A B$ a & $\mathrm{Aa}$ \\
\hline \multirow[t]{4}{*}{ Gel + EDTA } & 0 & 3 & 1 & 0 \\
\hline & 1 & 2 & 1 & 0 \\
\hline & 2 & 0 & 3 & 5 \\
\hline & & $\mathrm{AB} a$ & $A B a b$ & $A b$ \\
\hline \multirow[t]{4}{*}{ Solução de $\mathrm{NaOCl}$} & 0 & 4 & 4 & 1 \\
\hline & 1 & 6 & 6 & 8 \\
\hline & 2 & 0 & 0 & 1 \\
\hline & & $A B a$ & $\mathrm{AB}$ a & $\mathrm{Ba}$ \\
\hline \multirow{4}{*}{$\begin{array}{l}\text { Solução de } \mathrm{NaOCl}+ \\
\text { EDTA }\end{array}$} & 0 & 8 & 5 & 1 \\
\hline & 1 & 2 & 5 & 9 \\
\hline & 2 & 0 & 0 & 0 \\
\hline & & $\mathrm{Aa}$ & $\mathrm{B} a \mathrm{~b}$ & $\mathrm{BC} b$ \\
\hline \multirow[t]{4}{*}{ Gel de $\mathrm{NaOCl}$} & 0 & 0 & 0 & 0 \\
\hline & 1 & 7 & 4 & 1 \\
\hline & 2 & 3 & 6 & 9 \\
\hline & & $\mathrm{B} \mathrm{a}$ & $A a b$ & $A b$ \\
\hline \multirow[t]{4}{*}{ Gel de $\mathrm{NaOCl}+$ EDTA } & 0 & 4 & 0 & 1 \\
\hline & 1 & 4 & 6 & 2 \\
\hline & 2 & 2 & 4 & 7 \\
\hline & & $A B$ a & $A B a b$ & $A B b$ \\
\hline
\end{tabular}


Figura 1. Micrografias obtidas por MEV dos três terços radiculares dos diferentes grupos experimentais.

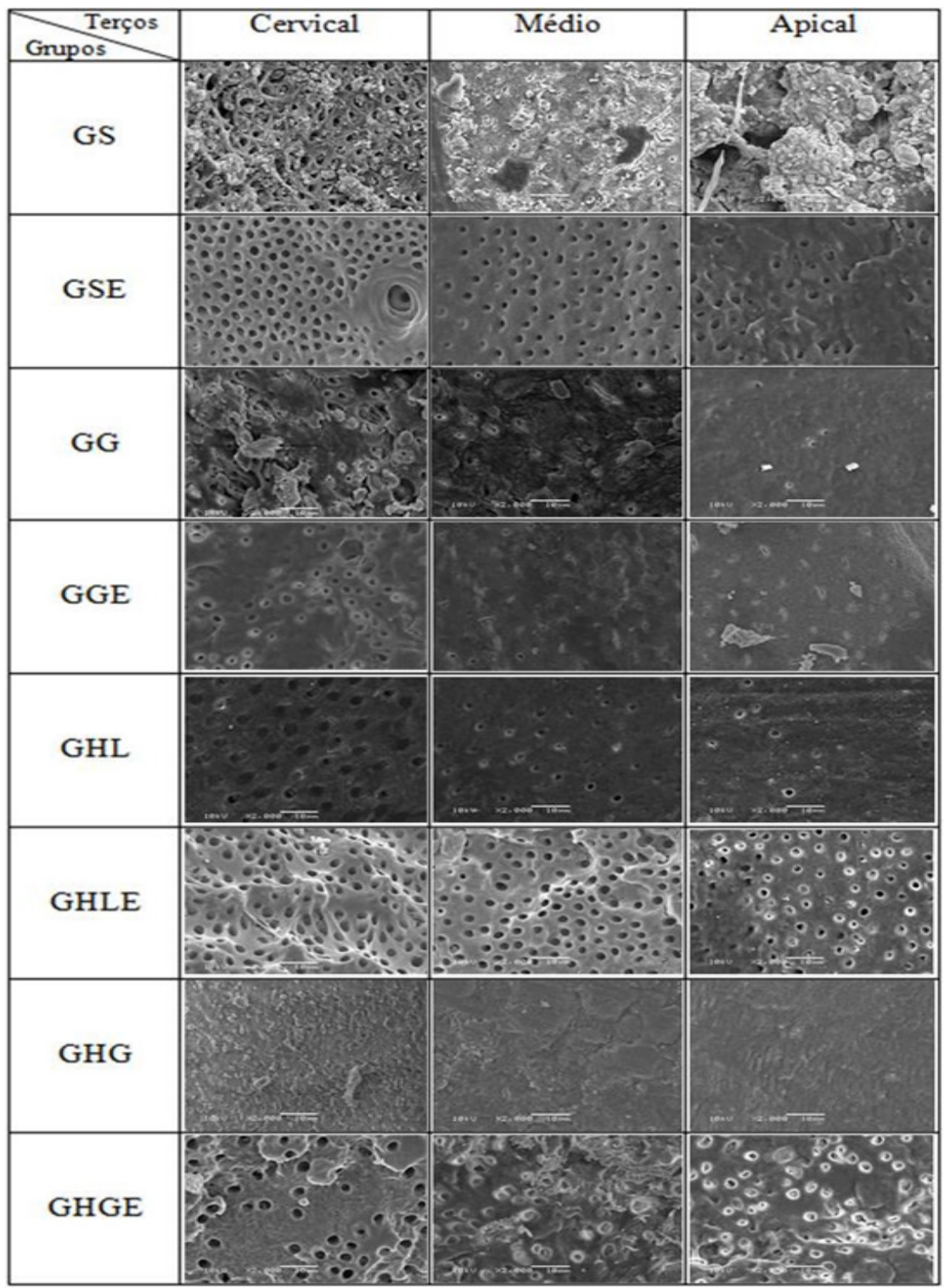

\section{DISCUSSÃO}

O uso do $\mathrm{NaOCl}$ gel pode ser eficaz na remoção da smear layer e ainda eliminar os efeitos colaterais da solução de hipoclorito de sódio9. A smear layer dificulta o contato da solução irrigadora com a superfície dentinária, afetando, negativamente, o processo de desinfecção do sistema de canais radiculares, além de reduzir os níveis de resistência de união dos materiais seladores à dentina e favorecer a microinfiltração coronária ${ }^{17}$. O gel de clorexidina, por exemplo, como auxiliar químico intracanal, no estudo de Gonçalves et al. (2014) ${ }^{18}$ mostrou ação antimicrobiana em microrganismos como Enterococcus faecalis e sua viscosidade produziu excelente ação conjunta no processo de limpeza mecânica do sistema de canais radiculares.

A hipótese nula de que não haveria diferença estatística na formação da smear layer promovida pelos auxiliares químicos testados foi rejeitada. No estudo, pode-se observar que a limpeza da parede dentinária, com a presença de mais túbulos dentinários abertos, foi melhor quando da utilização do soro fisiológico e do $\mathrm{NaOCl}$ líquido como substâncias irrigadoras, ambos associados à irrigação final com EDTA. Essa constatação também foi obtida no estudo de Menezes et al. $(2003)^{19}$. Por outro lado, os grupos que utilizaram o $\mathrm{NaOCl}$ na forma de gel e controle de base gel, ambos sem uso do EDTA, demonstraram os resultados com maior frequência de escores elevados para a presença de smear layer. Nascimento et al. (2015) observaram que as substâncias irrigadoras na forma de gel testadas $(\mathrm{NaOCl}$ a $5 \%$, Clorexidina a $2 \%$ e EDTA a $24 \%$ ) permaneceram aderidas às paredes do canal 20 .

No estudo de Zand et al. (2010), não se verificou diferença entre os grupos de $\mathrm{NaOCl}$ na forma de gel e líquido, ambos com uso de EDTA ${ }^{9}$. A associação do $\mathrm{NaOCl}$ gel e EDTA, no nosso estudo, apresentou um resultado melhor que sem a associação desse quelante, embora sem diferença estatística. Os túbulos 
dentinários ainda apresentavam resíduos sobre a sua superfície.

Comparando a mesma substância irrigadora, com e sem associação do quelante, pode-se verificar que os melhores resultados nos três terços radiculares de limpeza da parede dentinária e presença de túbulos dentinários abertos foram nos grupos que utilizaram o EDTA. Esses achados estão de acordo com os obtidos nos estudos de Yamashita et al. $\left(2003^{121} \mathrm{e}\right.$ Vasconcelos et al. (2007) ${ }^{22}$.

A associação da substância irrigadora que, no caso do estudo, é o $\mathrm{NaOCl}$, ao EDTA tende a promover um maior poder de limpeza nas paredes dentinárias durante o preparo pelo fato de a substância irrigadora ser um agente halógeno e do ácido etilenodiamino tetra-acético ser um quelante; combinação que promove uma ação mais eficaz nas porções orgânicas e inorgânicas da smear layer.

Na comparação da limpeza dentinária entre os três terços radiculares, pode-se verificar que o terço cervical apresentou os melhores resultados em todos os grupos testados quando comparados ao terço apical do canal, assim como nos estudos de Yamashita et al. (2003) $)^{21}$, Carvalho et al. (2008) ${ }^{23}$ e Kumar et al. $(2015)^{24}$. Somente o estudo de Mônika e Fröner $(2006)^{25}$ não observou diferença entre os três terços radiculares.

Segundo Carvalho et al. (2008)23, a obtenção de um melhor poder de limpeza no terço cervical, seguido do médio e apical deve-se à facilidade de execução do procedimento de irrigação e aspiração; somado a isso, um maior volume de substância irrigadora que circula nessa região mais cervical do canal radicular

\section{CONCLUSÕES}

De acordo com o estudo, independentemente dos auxiliares químicos testados, a associação do toalete final com EDTA promoveu uma maior limpeza da parede dentinária. O soro fisiológico e o $\mathrm{NaOCl}$ líquido a 2,5\%, associados ao EDTA, apresentaram os resultados mais favoráveis para a limpeza das paredes do canal radicular. Independentemente do auxiliar químico, houve uma melhor capacidade de remoção da smear layer no terço cervical em relação ao apical.

\section{REFERÊNCIAS}

1. Machado M, Nabeshima CK, Leonardo MF, Reis FA, Britto ML, Cai S. Influence of reciprocating single-file and rotary instrumentation on bacterial reduction on infected root canals. Int Endod J. 2013 Nov; 46(11): 1083-7. doi: 10.1111/ iej. 12108

2. Raut AW, Mantri V, Palekar A, Gadodia R, Kala S, Raut RA. Comparative analysis of cleaning ability of three nickel-titanium rotary systems: protaper universal, k3 and mtwo: an in vitro scanning electron microscopic study. Niger Postgrad Med J. 2016 Oct-Dec; 23(4):221-6. doi: 10.4103/1117-1936.

3. Naenni N, Thoma K, Zehnder M. Soft tissue dissolution capacity of currently used and potential endodontic irrigants. J Endod. 2004 Nov; 30(11):785-7.

4. Peters OA, Laib A, Göhring TN, Barbakow F. Changes in root canal geometry after preparation assessed by high-resolution computed tomography. J Endod. 2001 Jan; 27(1):1-6

5. Poggio C, Arciola CR, Dagna A, Chiesa M, Sforza D, Visai L. Antimicrobial activity of sodium hypochlorite-based irrigating solutions. Int J Artif Organs. 2010 Sep; 33(9):654-9.

6. Zehnder M. Root canal irrigants. J Endod. 2006 May; 32(5):389-98.

7. Jayasimha RU, Mylswamy S. The Effect of $4 \%$ Lignocaine gel, $5 \%$ Amiloride $\mathrm{HCl}$ and 10\% Chlorpromazine on E.faecalis. J Conserv Dent. 2011 Apr; 14(2):160-3. doi: 10.4103/0972-0707.82623.

8. Pashley EL, Birdsong NL, Bowman K, Pashley DH. Cytotoxic effects of $\mathrm{NaOCl}$ on vital tissue. J Endod. 1985 Dec;11(12):525-8. doi: 10.1016/S00992399(85)80197-7.

9. Zand V, Lotfi M, Rahimi S, Mokhtari H, Kazemi A, Sakhamanesh V. A comparative scanning electron microscopic investigation of the smear layer after the use of sodium hypochlorite gel and solution forms as root canal irrigants. J Endod. 2010 Jul; 36(7): 1234-7. doi: 10.1016/j.joen.2010.02.033.

10. Bosch-Aranda ML, Canalda-Sahli C, Figueiredo R, Gay-Escoda C. Complications following an accidental sodium hypochlorite extrusion: a report of two cases. J Clin Exp Dent. 2012 Jul; 4(3):e194-e198. doi: 10.4317/jced.50767.
11. Kandian S, Chander S, Bishop K. Management of sodium hypochlorite extrusion beyond the root apex during root canal treatment: a case report. Prim Dent J. 2014 Feb; 3(1):72-5. doi: 10.1308/205016814812135805.

12. Guivarc'h M, Ordioni U, Ahmed HM, Cohen S, Catherine JH, Bukiet F. Sodium Hypochlorite Accident: A Systematic Review. J Endod. 2017 Jan; 43(1):16-24. doi: 10.1016/j.joen.2016.09.023.

13. Kleier DJ, Averbach RE, Mehdipour O. The sodium hypochlorite accident: experience of diplomates of the american board of endodontics. J Endod. 2008 Nov; 34(11):1346-50. doi: 10.1016/j.joen.2008.07.02.

14. Ferraz CC, Gomes BP, Zaia AA, Teixeira FB, Souza-Filho FJ. In vitro assessment of the antimicrobial action and the mechanical ability of chlorhexidine gel as an endodontic irrigant. J Endod. 2001 Jul; 27(7):452-5.

15. Ahmetoglu F, Keles A, Yalcin M, Simsek N. Effectiveness of different irrigation systems on smear layer removal: A scanning electron microscopic study. Eur J Dent. 2014 Jan-Mar; 8(1):53-7. doi: 10.4103/1305-7456.126241.

16. Torabinejad M, Khademi AA, Babagoli J, Cho Y, Johnson WB, Bozhilov K, Kim J, Shabahang S. A new solution for the removal of the smear layer. J endod. 2003 Mar; 29(3):170-5. doi:10.1097/00004770-200303000-00002.

17. Prado M, Assis DF, Simão RA. Efeito de diferentes soluções utilizadas como irrigante final na superfície dentinária: análise de rugosidade. Rev Odontol UNESP. 2014; 43(1):36-40. doi: http://dx.doi.org/10.1590/S180725772014000100006

18. Gonçalves L, Silva-Sousa YT, Raucci Neto W, Teixeira CS, Sousa-Neto MD, Alfredo $\mathrm{E}$. Effect of different irrigation protocols on the radicular dentin interface and bond strength with a metacrylate-based endodontic sealer. Microsc Res Tech. 2014 Jun; 77(6):446-52. doi: 10.1002/jemt.22365.

19. Menezes AC, Zanet CG, Valera MC. Smear layer removal capacity of disinfectant solutions used with and without edta for the irrigation of canals: a sem study. Pesqui Odontol Bras. 2003 Out-Dez;17(4): 349-55. doi: http://dx.doi. org/10.1590/S1517-74912003000400010. 
20. Nascimento AL, Busanello FH, Só MV, Kuga MC, Pereira JR, Grecca FS. Residues of different gel formulations on dentinal walls: a sem/eds analysis. Microsc Res Tech. 2015; 78(6):495-9. doi: 10.1002/jemt.22500.

21. Yamashita JC, Tanomaru Filho M, Leonardo MR, Rossi MA, Silva LA. Scanning electron microscopic study of the cleaning ability of chlorhexidine as a rootcanal irrigant. Int Endod J. 2003 Jun; 36(6):391-4

22. Vasconcelos BC, Luna-Cruz SM, De-Deus G, Moraes IG, Maniglia-Ferreira C, Gurgel-Filho ED. Cleaning ability of chlorhexidine gel and sodium hypochlorite associated or not with EDTA as root canal irrigants: a scanning electron microscopy study. J Appl Oral Sci. 2007 Oct;15(5): 387-91.
23. Carvalho AS, Camargo CH, Valera MC, Camargo SE, Mancini MN. Smear layer removal by auxiliary chemical substances in biomechanical preparation: a scanning electron microscope study. J Endod. 2008 Nov; 34(11):1396-1400. doi: 10.1016/j.joen.2008.08.012.

24. Kumar P, Prasad N, Darawade A, Bhagat SK, Narayana N, Darawade P. The effect of four commonly used root canal irrigants on the removal of smear layer: an in-vitro scanning electron microscope study. J Int Oral Health. 2015 Sep; 7(9):88-93.

25. Mônika CM, Fröner IC. A scanning electron microscopic evaluation of different root canal irrigation regimens. Braz Oral Res. 2006 Jul-Sep; 20(3): 23540. 